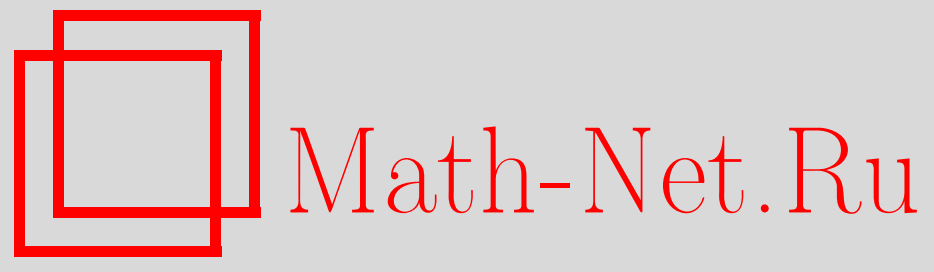

Р. А. Бандалиев, Двухвесовые неравенства для сверточных операторов в пространстве Лебега, Матем. заметкu, 2006, том 80, выпуск 1, 3-10

DOI: https://doi.org/10.4213/mzm2773

Использование Общероссийского математического портала Math-Net.Ru подразумевает, что вы прочитали и согласны с пользовательским соглашением http://www . mathnet.ru/rus/agreement

Параметры загрузки:

IP : 3.85 .73 .92

26 апреля 2023 г., 15:33:04

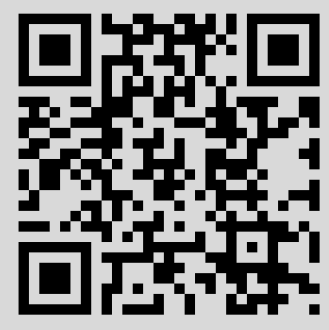


УДК 517.518

\section{ДВУХВЕСОВЫЕ НЕРАВЕНСТВА ДЛЯ СВЕРТОЧНЫХ ОПЕРАТОРОВ В ПРОСТРАНСТВЕ ЛЕБЕГА}

\section{Р. А. Бандалиев}

В работе доказана теорема об ограниченности оператора свертки в весовом пространстве Лебега с ядром, удовлетворяющим некоторому варианту условия Хёрмандера.

Библиография: 13 названий.

Пусть $\mathbb{R}^{n}$ - $n$-мерное евклидово пространство точек $x=\left(x_{1}, \ldots, x_{n}\right), n \in \mathbb{N}$, где $\mathbb{N}$ - множество натуральных чисел, $\mathbb{R}_{0}^{n}=\mathbb{R}^{n} \backslash\{0\}, \quad|x|=\left(\sum_{i=1}^{n} x_{i}^{2}\right)^{1 / 2}$ и $(x, y)=$ $\sum_{i=1}^{n} x_{i} y_{i}$

Предположим, что $\omega$ - положительная, измеримая и вещественная функция, заданная в $\mathbb{R}^{n}$, т.е. является весовой функцией. Через $L_{p, \omega}\left(\mathbb{R}^{n}\right)$ будем обозначать пространство измеримых на $\mathbb{R}^{n}$ функций $f(x)$, для которых конечна норма

$$
\|f\|_{L_{p, \omega}\left(\mathbb{R}^{n}\right)}=\left(\int_{\mathbb{R}^{n}}|f(x)|^{p} \omega(x) d x\right)^{1 / p}, \quad 1 \leqslant p<\infty .
$$

В случае, когда $p=\infty$, норма в пространстве $L_{\infty, \omega}\left(\mathbb{R}^{n}\right)$ определяется следующим образом:

$$
\|f\|_{L_{\infty}, \omega}\left(\mathbb{R}^{n}\right)=\|f\|_{L_{\infty}\left(\mathbb{R}^{n}\right)}=\operatorname{ess~sup}_{x \in \mathbb{R}^{n}}|f(x)| .
$$

При $\omega=1$ получается невесовое пространство $L_{p}$, т.е. $L_{p, 1}\left(\mathbb{R}^{n}\right)=L_{p}\left(\mathbb{R}^{n}\right)$.

Говорят, что $f \in L_{p}^{\text {loc }}\left(\mathbb{R}^{n}\right), 1 \leqslant p<\infty$, если $f \in L_{p}(F)$ на любом замкнутом ограниченном множестве $F \subset \mathbb{R}^{n}$.

Предположим, что $K: \mathbb{R}_{0}^{n} \rightarrow \mathbb{R}, K \in L_{1}^{\text {loc }}\left(\mathbb{R}_{0}^{n}\right)$ - некоторая функция, удовлетворяющая условиям

1) $K(t x) \equiv K\left(t x_{1}, \ldots, t x_{n}\right)=t^{-n} K(x)$ для любых $t>0, x \in \mathbb{R}_{0}^{n}$;

2) $\int_{|x|=1} K(x) d \sigma(x)=0$

3) $\int_{0}^{1} \frac{w(t)}{t} d t<\infty$, где $w(t)=\sup _{|\xi-\eta| \leqslant t}|K(\xi)-K(\eta)|$ при $|\xi|=|\eta|=1$.

Для функции $f \in L_{p}\left(\mathbb{R}^{n}\right), 1<p<\infty$, рассмотрим следующий сингулярный интеграл:

$$
T f(x)=\lim _{\varepsilon \rightarrow 0+} \int_{|x-y|>\varepsilon} K(x-y) f(y) d y=\text { p.v. } \int_{\mathbb{R}^{n}} K(x-y) f(y) d y,
$$

где последний интеграл понимается в смысле главного значения и $\varepsilon>0$ - некоторое число.

Имеет место следующая теорема.

(C) Р. А. БАндАлиЕв, 2006 
ТеОРема 1 [1]. Пусть ядро К сингулярного интеграла (1) удовлетворяет условиям 1)-3). Предположим, что $f \in L_{p}\left(\mathbb{R}^{n}\right), 1<p<\infty$. Тогда сингулярный интеграл существует для п.в. $x \in \mathbb{R}^{n}$ и имеет место неравенство

$$
\|T f\|_{L_{p}\left(\mathbb{R}^{n}\right)} \leqslant C\|f\|_{L_{p}\left(\mathbb{R}^{n}\right)},
$$

где $C>0$ не зависит от $f$.

Эта теорема была доказана в работе Кальдерона-Зигмунда [1] и носит название теоремы Кальдерона-Зигмунда.

Далее, в работе [2] Хёрмандер, наложив на ядро сингулярного интеграла (1) более слабое ограничение, а именно,

$$
\int_{|x|>2|y|}|K(x-y)-K(x)| d x \leqslant C,
$$

где $K \in L_{1}^{\text {loc }}\left(\mathbb{R}_{0}^{n}\right), \quad C>0$ - постоянная, не зависящая от $y$, заменяя условие 3$)$ на условие (2), при выполнении условий 1), 2) доказал теорему 1 для сингулярных интегралов с ядрами, удовлетворяющими условию (2). Это условие связано с условием 3) и при выполнении этого условия имеет место неравенство (2) (см. [3]).

С другой стороны, сингулярные интегралы, ядра которых не удовлетворяют условию Хёрмандера (2), рассматриваются обширно (например, осцилляционные и другие сингулярные интегралы) (см. [4]).

Предположим, что $K \in L_{2}\left(\mathbb{R}^{n}\right)$ - некоторая функция, удовлетворяющая следующим условиям:

$(K 1)\|\widehat{K}\|_{\infty} \leqslant C$;

$(K 2)|K(x)| \leqslant \frac{C}{|x|^{n}}$

$(K 3)$ существуют функции $A_{1}, \ldots, A_{m} \in L_{1}^{\text {loc }}\left(\mathbb{R}_{0}^{n}\right)$ и $\Phi=\left\{\phi_{1}, \ldots, \phi_{m}\right\}$ - конечное семейство существенно ограниченных функций в $\mathbb{R}^{n}$ таких, что $\left.\mid \operatorname{det}\left[\phi_{j}\left(y_{i}\right)\right)\right]\left.\right|^{2} \in$ $R H_{\infty}\left(\mathbb{R}^{n m}\right), y_{i} \in \mathbb{R}^{n}, i, j=1, \ldots, m ;$

$(K 4)$ для фиксированного $\gamma>0$ и для любого $|x|>2|y|>0$,

$$
\left|K(x-y)-\sum_{i=1}^{m} A_{i}(x) \phi_{i}(y)\right| \leqslant C \frac{|y|^{\gamma}}{|x-y|^{n+\gamma}},
$$

где $C>0$ - некоторая постоянная, $\widehat{K}(\xi)=\int_{\mathbb{R}^{n}} e^{-i(x, \xi)} K(x) d x$ - преобразование Фурье функции $K$. Вообще говоря, функции $A_{i}, \phi_{i}, i=1, \ldots, m$, определенные в $\mathbb{R}_{0}^{n}$, являются комплекснозначными.

ЗАмЕЧАниЕ 1. Отметим, что каждое ядро, удовлетворяющее условию (3), удовлетворяет также условию

$$
\int_{|x|>2|y|}\left|K(x-y)-\sum_{i=1}^{m} A_{i}(x) \phi_{i}(y)\right| d x \leqslant C \quad \text { при } \quad|x|>2|y| .
$$

Действительно, интегрируя обе части неравенства (3) по множеству $|x|>2|y|$, используя неравенство $|x-y| \geqslant|x| / 2$ и переходя к сферическим координатам, получим

$$
\int_{|x|>2|y|}\left|K(x-y)-\sum_{i=1}^{m} A_{i}(x) \phi_{i}(y)\right| d x \leqslant C|y|^{\gamma} \int_{2|y|}^{\infty} \frac{d t}{t^{1+\gamma}}=C .
$$


Поэтому условие (4) является более слабым ограничением на ядро $K$, чем условие (3).

Отметим, что условия $(K 1)-(K 4)$ были наложены в работе [4], а условие (4) было рассмотрено в работе [5]. Например, при $m=1, A_{1}(x)=K(x), \phi_{1}(y) \equiv 1$ из условия (4) получается условие Хёрмандера (2). Отметим, что в этом смысле условие (4) является обобщением условия Хёрмандера (2).

Существуют еще другие, а именно, условия, которые сильнее, чем условие (2) (cм. [6], [7]).

ПримеР. Рассмотрим ядро

$$
K(x)=\frac{\sin x}{x}, \quad x \in \mathbb{R}_{0} .
$$

Эта функция удовлетворяет условиям $(K 1)-(K 4)$. Действительно, так как

$$
\widehat{K}(\xi)= \begin{cases}2 \pi, & |\xi| \leqslant 1 \\ \pi, & \xi= \pm 1 \\ 0, & |\xi|>1\end{cases}
$$

получим, что $\|K\|_{\infty}=2 \pi$ и условие $(K 1)$ выполняется. Очевидно, что $|\sin x / x| \leqslant$ $1 /|x|$.

Теперь покажем, что выполняется условие $(K 3)$. Используя равенство $\sin x=$ $\left(e^{i x}-e^{-i x}\right) /(2 i)$, выберем функции $A_{j}$ и $\phi_{j}, j=1,2$, следующим образом:

$$
A_{1}(x)=\frac{e^{i x}}{2 i x}, \quad A_{2}(x)=-\frac{e^{-i x}}{2 i x}, \quad \phi_{1}(y)=e^{-i y}, \quad \phi_{2}(y)=e^{i y}, \quad m=2 .
$$

Заметим, что $\left|A_{1}(x)\right|=\left|A_{2}(x)\right|=1 /(2|x|) \in L_{1}^{\text {loc }}\left(\mathbb{R}_{0}\right)$. Далее,

$$
\left.\operatorname{det}\left[\phi_{j}\left(y_{i}\right)\right)\right]=\left|\begin{array}{cc}
e^{-i y_{1}} & e^{-i y_{2}} \\
e^{i y_{1}} & e^{i y_{2}}
\end{array}\right|=e^{-i\left(y_{1}-y_{2}\right)}-e^{i\left(y_{1}-y_{2}\right)}
$$

где $y_{1}, y_{2} \in \mathbb{R}$. Тогда $\left.\mid \operatorname{det}\left[\phi_{j}\left(y_{i}\right)\right)\right]\left.\right|^{2}=\left|1-e^{2 i\left(y_{1}-y_{2}\right)}\right|=4 \sin ^{2}\left(y_{1}-y_{2}\right)$ и, применяя теорему о среднем значении, вычислим следующий интеграл:

$$
\frac{1}{|B|} \int_{B} \sin ^{2}\left(y_{1}-y_{2}\right) d y_{1} d y_{2}=C \geqslant C \sup _{B} \sin ^{2}\left(y_{1}-y_{2}\right)
$$

где $B$ - произвольный шар в $\mathbb{R}^{2}$ с центром в начале координат, $\left(y_{1}, y_{2}\right) \in B$ и $0 \leqslant$ $C \leqslant 1$. А это и означает, что $4 \sin ^{2}\left(y_{1}-y_{2}\right) \in R H_{\infty}\left(\mathbb{R}^{2}\right)$. Теперь проверим условие $(K 4)$. Так как $|x|>2|y|$, то $|x-y| \leqslant|x|+|y| \leqslant \frac{3}{2}|x|$. Поэтому имеем

$$
\left|\frac{\sin (x-y)}{x-y}-\sum_{i=1}^{m} A_{i}(x) \phi_{i}(y)\right| d x=|\sin (x-y)|\left|\frac{1}{x-y}-\frac{1}{x}\right| \leqslant \frac{|y|}{|x||x-y|} \leqslant \frac{2}{3} \frac{|y|}{|x-y|^{2}},
$$

т.е. при $\gamma=1$ условие $(K 4)$ выполняется.

Но функция $K(x)=\sin x / x$ не удовлетворяет условиям 1$), 2)$ и условию Хёрмандера (2). 
ОПРЕДЕЛЕНИЕ 1 [4]. Говорят, что положительная, измеримая и локально суммируемая функция удовлетворяет обратному условию Гёлъдера $R H_{\infty}$, или $g \in$ $R H_{\infty}\left(\mathbb{R}^{n}\right)$, если

$$
0<\sup _{x \in B} g(x) \leqslant C \frac{1}{|B|} \int_{B} g(x) d x,
$$

где $B$ - произвольный шар с центром в нуле и $C>0$ - постоянная, не зависящая от $B$.

ОПРЕДЕЛЕНИЕ 2 [8]. Говорят, что локально интегрируемая весовая функция $\nu$ принадлежит $A_{p}\left(\mathbb{R}^{n}\right), 1<p<\infty$, если

$$
\sup _{B}\left(|B|^{-1} \int_{B} \nu(x) d x\right)\left(|B|^{-1} \int_{B} \nu(x)^{1-p^{\prime}} d x\right)^{p-1}<\infty
$$

где супремум берется по всем шарам $B \subset \mathbb{R}^{n}$ и $p^{\prime}=p /(p-1)$.

ЗАмечание 2 . Отметим, что из условия $R H_{\infty}\left(\mathbb{R}^{n}\right)$ следует хорошо известное обратное неравенство Гёльдера:

$$
\left(\frac{1}{|B|} \int_{B} g(x)^{1+\varepsilon} d x\right)^{\frac{1}{1+\varepsilon}} \leqslant C\left(\int_{B} g(x) d x\right)
$$

для любого $\varepsilon>0$. А это условие, как известно, характеризует условие $A_{p}\left(\mathbb{R}^{n}\right)$ (см. [6; c. 403]).

Предположим, что функция $K$ удовлетворяет условиям $(K 1)-(K 4)$. Для $f \in$ $L_{p}\left(\mathbb{R}^{n}\right)$ определим сверточный оператор, порожденный ядром $K$ :

$$
A f(x)=\int_{\mathbb{R}^{n}} K(x-y) f(y) d y .
$$

Для сверточного оператора (5) доказана следующая теорема.

Теорема 2 [4]. Пусть $1<p<\infty, w \in A_{p}\left(\mathbb{R}^{n}\right)$ и предположим, что ядро сверточного оператора (5) удовлетворяет условиям $(K 1)-(K 4)$. Тогда имеет место неравенство

$$
\|T f\|_{L_{p, w}\left(\mathbb{R}^{n}\right)} \leqslant C\|f\|_{L_{p, w}\left(\mathbb{R}^{n}\right)},
$$

где $C>0$ не зависит от $f$.

Отметим, что в невесовом случае при отсутствии условия $(K 2)$ и если заменить условие (3) на условие (4), теорема 2 была доказана в [5].

Имеет место

Теорема 3. Пусть $1<p<\infty, u(t) u v(t)$ - положительные функиии, определенные на $(0, \infty)$.

1. Для справедливости неравенства

$$
\int_{0}^{\infty} u(t)\left|\int_{0}^{t} \varphi(\tau) d \tau\right|^{p} d t \leqslant C_{1} \int_{0}^{\infty} v(t)|\varphi(t)|^{p} d t
$$


с постоянной $C_{1}$, не зависящей от $\varphi$, необходимо и достаточно выполнения условия

$$
\sup _{t>0}\left(\int_{t}^{\infty} u(\tau) d \tau\right)\left(\int_{0}^{t} v(\tau)^{1-p^{\prime}} d \tau\right)^{p-1}<\infty .
$$

2. Для справедливости неравенства

$$
\int_{0}^{\infty} u(t)\left|\int_{t}^{\infty} \varphi(\tau) d \tau\right|^{p} d t \leqslant C_{2} \int_{0}^{\infty} v(t)|\varphi(t)|^{p} d t
$$

c постоянной $C_{2}$, не зависящей от $\varphi$, необходимо и достаточно выполнения условия

$$
\sup _{t>0}\left(\int_{0}^{t} u(\tau) d \tau\right)\left(\int_{t}^{\infty} v(\tau)^{1-p^{\prime}} d \tau\right)^{p-1}<\infty .
$$

Отметим, что теорема 3 была доказана в работах [9]-[11].

Лемма 1. Пусть $1 \leqslant p<\infty, \beta \geqslant 1, \varphi \in A_{p}\left(\mathbb{R}^{n}\right)-$ радиальная функиия, т.е. $\varphi(x)=\varphi(|x|)$, и пусть $u, u_{1}$ - положительные неубывающие (невозрастающие) функиии, определенные на $(0, \infty)$. Предположим, что $\omega=u \varphi, \omega_{1}=u_{1} \varphi$ и весовая пара $\left(\omega, \omega_{1}\right)$ удовлетворяет следующему условию:

$$
\begin{gathered}
\sup _{t>0}\left(\int_{t}^{\infty} \omega_{1}(\tau) \tau^{-1-n(p-1)} d \tau\right)\left(\int_{0}^{t / 2} \omega(\tau)^{1-p^{\prime}} \tau^{n-1} d \tau\right)^{p-1}<\infty \\
\left(\sup _{t>0}\left(\int_{0}^{t / 2} \omega_{1}(\tau) \tau^{n-1} d \tau\right)\left(\int_{t}^{\infty} \omega(\tau)^{1-p^{\prime}} \tau^{-1-n\left(p^{\prime}-1\right)} d \tau\right)^{p-1}<\infty\right) .
\end{gathered}
$$

Тогда существует положительная постоянная $C$ такая, что для любого $t>0$ имеет место следующее неравенство:

$$
u_{1}(\beta t) \leqslant C u(t) \quad\left(u_{1}\left(\frac{t}{\beta}\right) \leqslant C u(t)\right) .
$$

Лемма 1 доказана в работе [12].

\section{Основные результаты.}

Теорема 4. Пусть $1<p<\infty$, ядро $K$ сверточного оператора (5) удовлетворяет условиям $(K 1)-(K 4)$. Предположим, что и, $u_{1}$ - положительные неубывающие функиии на $(0, \infty), \varphi \in A_{p}\left(\mathbb{R}^{n}\right)$ - радиальная функиия, $\omega=u \varphi и \omega_{1}=u_{1} \varphi$.

Если весовая пара $\left(\omega_{1}, \omega\right)$ удовлетворяет условию (8), то существует $C>0$ такое, что для любого $f \in L_{p, \omega}\left(\mathbb{R}^{n}\right)$ имеет место неравенство

$$
\int_{\mathbb{R}^{n}}|T f(x)|^{p} \omega_{1}(|x|) d x \leqslant C \int_{\mathbb{R}^{n}}|f(x)|^{p} \omega(|x|) d x .
$$

ДокАЗАТЕЛЬство. Неравенство (10) сначала доказывается для тех неубывающих функций, для которых имеет место следующее представление:

$$
u_{1}(t)=u_{1}(0)+\int_{0}^{t} \psi(\tau) d \tau
$$

где $u_{1}(0)=\lim _{t \rightarrow 0+} u_{1}(t)$ и $\psi(t)-$ неотрицательная функция на $(0, \infty)$. 
Действительно, для неубывающих на $(0,+\infty)$ функций $u_{1}(t)$ существует последовательность абсолютно непрерывных функций $\varphi_{n}(t)$ таких, что $\lim _{n \rightarrow \infty} \varphi_{n}(t)=u_{1}(t)$ п.в., $\varphi_{n}(t) \leqslant u_{1}(t), \varphi_{n}(0)=u_{1}(0)$, функции $\varphi_{n}(t)$ не убывают и, кроме того, $\varphi_{n}(t)=\int_{0}^{t} \psi_{n}(\tau) d \tau+\varphi_{n}(0)$, где $\psi_{n}(t)$ - неотрицательные функции на $(0, \infty)$. Отсюда с применением теоремы Фату получается справедливость оценки (10) для неубывающих функций (см. [12], [13]).

Оценим левую сторону неравенства (10). Имеем

$$
\begin{aligned}
\int_{\mathbb{R}^{n}}|T f(x)|^{p} \omega_{1}(|x|) d x=\int_{\mathbb{R}^{n}}|T f(x)|^{p} \varphi(|x|)\left(u_{1}(0)+\int_{0}^{|x|} \psi(t) d t\right) d x \\
=\int_{\mathbb{R}^{n}}|T f(x)|^{p} \varphi(|x|) u_{1}(0) d x+\int_{\mathbb{R}^{n}} \varphi(|x|)\left(\int_{0}^{|x|}|T f(x)|^{p} \psi(t) d t\right) d x=D_{1}+D_{2} .
\end{aligned}
$$

Если $u_{1}(0)=0$, то $D_{1}=0$. Однако если $u_{1}(0)>0$, то по теореме 2 имеем

$$
\begin{aligned}
D_{1} & =u_{1}(0) \int_{\mathbb{R}^{n}}|T f(x)|^{p} \varphi(|x|) d x \leqslant C_{1} u_{1}(0) \int_{\mathbb{R}^{n}}|f(x)|^{p} \varphi(|x|) d x \\
& \leqslant C_{1} \int_{\mathbb{R}^{n}}|f(x)|^{p} \varphi(|x|) u_{1}(|x|) d x \leqslant C_{2} \int_{\mathbb{R}^{n}}|f(x)|^{p} \varphi(|x|) u(|x|) d x \\
& =C_{2} \int_{\mathbb{R}^{n}}|f(x)|^{p} \omega(|x|) d x .
\end{aligned}
$$

Оценим $D_{2}$. Используя теорему Фубини, получим

$$
\begin{aligned}
D_{2}= & \int_{\mathbb{R}^{n}}|T f(x)|^{p} \varphi(|x|)\left(\int_{0}^{|x|} \psi(t) d t\right) d x=\int_{0}^{\infty} \psi(t)\left(\int_{|x|>t}|T f(x)|^{p} \varphi(|x|) d x\right) d t \\
\leqslant & C_{3} \int_{0}^{\infty} \psi(t)\left(\int_{|x|>t}\left|\int_{|y|>\frac{t}{2}} K(x-y) f(y) d y\right|^{p} \varphi(|x|) d x\right) d t \\
& \quad+C_{3} \int_{0}^{\infty} \psi(t)\left(\int_{|x|>t}\left|\int_{|y| \leqslant \frac{t}{2}} K(x-y) f(y) d y\right|^{p} \varphi(|x|) d x\right) d t=D_{21}+D_{22}
\end{aligned}
$$

Снова используя теорему 2, лемму 1 и теорему Фубини, получим

$$
\begin{aligned}
D_{21} & \leqslant C_{3} \int_{0}^{\infty} \psi(t)\left(\int_{\mathbb{R}^{n}}\left|\int_{\mathbb{R}^{n}} K(x-y) f(y) \chi_{\left\{z:|z|>\frac{t}{2}\right\}}(y) d y\right|^{p} \varphi(|x|) d x\right) d t \\
& \leqslant C_{4} \int_{0}^{\infty} \psi(t)\left(\int_{|x|>\frac{t}{2}}|f(x)|^{p} \varphi(|x|) d x\right) d t \\
& =C_{4} \int_{\mathbb{R}^{n}}|f(x)|^{p} \varphi(|x|)\left(\int_{0}^{2|x|} \psi(t) d t\right) d x \\
& \leqslant C_{4} \int_{\mathbb{R}^{n}}|f(x)|^{p} \varphi(|x|) u_{1}(2|x|) d x \leqslant C_{5} \int_{\mathbb{R}^{n}}|f(x)|^{p} \varphi(|x|) u(|x|) d x \\
& =C_{5} \int_{\mathbb{R}^{n}}|f(x)|^{p} \omega(|x|) d x .
\end{aligned}
$$


Оценим теперь $D_{22}$. Если $|x|>t$ и $|y| \leqslant t / 2,|x-y| \geqslant|x|-|y| \geqslant|x|-t / 2=|x| / 2$, то

$$
\begin{aligned}
D_{22} & \leqslant C_{6} \int_{0}^{\infty} \psi(t)\left(\int_{|x|>t} \varphi(|x|)\left(\int_{|y| \leqslant \frac{2}{2}} \frac{|f(y)|}{|x-y|^{n}} d y\right)^{p} d x\right) d t \\
& \leqslant C_{7} \int_{0}^{\infty} \psi(t)\left(\int_{|x|>t} \frac{\varphi(|x|)}{|x|^{n p}} d x\right)\left(\int_{|y| \leqslant \frac{t}{2}}|f(y)| d y\right)^{p} d t \\
& \leqslant C_{7} \int_{0}^{\infty} \psi(2 s)\left(\int_{|x|>2 s} \frac{\varphi(|x|)}{|x|^{n p}} d x\right)\left(\int_{|y| \leqslant s}|f(y)| d y\right)^{p} d s \\
& =C_{8} \int_{0}^{\infty} \psi(2 s)\left(\int_{2 s}^{\infty} \varphi(\tau) \tau^{n-n p-1} d \tau\right)\left(\int_{0}^{s} t^{n-1}\left(\int_{|y|=1}|f(t \bar{y})| d \sigma(\bar{y})\right) d t\right)^{p} d s
\end{aligned}
$$

Кроме того, имеют место следующие оценки:

$$
\begin{aligned}
\int_{t}^{\infty} \psi(2 s) & \left(\int_{2 s}^{\infty} \varphi(\tau) \tau^{n-n p-1} d \tau\right) d s \leqslant \int_{2 t}^{\infty} \psi(s)\left(\int_{s}^{\infty} \varphi(\tau) \tau^{n-n p-1} d \tau\right) d s \\
= & \int_{2 t}^{\infty} \varphi(\tau) \tau^{n-n p-1}\left(\int_{2 t}^{\tau} \psi(s) d s\right) d \tau \leqslant \int_{2 t}^{\infty} \varphi(\tau) u_{1}(\tau) \tau^{n-n p-1} d \tau \\
\leqslant & \int_{t}^{\infty} \omega_{1}(\tau) \tau^{n-n p-1} d \tau
\end{aligned}
$$

Поэтому имеем

$$
\begin{aligned}
\sup _{t>0}\left(\int_{t}^{\infty}\right. & \left.\psi(2 s)\left(\int_{2 s}^{\infty} \varphi(\tau) \tau^{n-n p-1} d \tau\right) d s\right)\left(\int_{0}^{t / 2} \omega^{1-p^{\prime}}(\tau) \tau^{n-1} d \tau\right)^{p-1} \\
& \leqslant \sup _{t>0}\left(\int_{t}^{\infty} \omega_{1}(\tau) \tau^{n-n p-1} d \tau\right)\left(\int_{0}^{t / 2} \omega^{1-p^{\prime}}(\tau) \tau^{n-1} d \tau\right)^{p-1}<\infty
\end{aligned}
$$

Для величины $D_{22}$ в силу последнего неравенства из неравенства (6) получим

$$
\begin{aligned}
\int_{0}^{\infty} \psi(2 s) & \left(\int_{2 s}^{\infty} \varphi(\tau) \tau^{n-n p-1} d \tau\right)\left(\int_{0}^{s} t^{n-1}\left(\int_{|y|=1}|f(t \bar{y})| d \sigma(\bar{y})\right) d t\right)^{p} d s \\
\leqslant & C_{9} \int_{0}^{\infty} \omega(t) t^{n-1}\left(\int_{|y|=1}|f(t \bar{y})| d \sigma(\bar{y})\right)^{p} d t \\
\leqslant & C_{10} \int_{0}^{\infty} \omega(t) t^{n-1}\left(\int_{|y|=1}|f(t \bar{y})|^{p} d \sigma(\bar{y})\right) d t=C_{10} \int_{\mathbb{R}^{n}}|f(x)|^{p} \omega(|x|) d x
\end{aligned}
$$

Теорема доказана.

\section{Аналогично доказывается}

Теорема 5. Пусть $1<p<\infty$, ядро $K$ сверточного оператора (5) удовлетворяет условиям (K1)-(K4). Предположим, что $u, u_{1}$ - положительные невозрастающие функции на $(0, \infty), \varphi \in A_{p}\left(\mathbb{R}^{n}\right)-$ радиальная функиия, $\omega=u \varphi и \omega_{1}=u_{1} \varphi$.

Если весовая пара $\left(\omega_{1}, \omega\right)$ удовлетворяет условию $(9)$, то существует $C>0$ такое, что для любого $f \in L_{p, \omega}\left(\mathbb{R}^{n}\right)$ имеет место неравенство (10). 
ДокАзАтЕЛЬство. Неравенство (10) сначала доказывается для тех невозрастающих функций, для которых имеет место следующее представление:

$$
u_{1}(t)=u_{1}(+\infty)+\int_{t}^{\infty} \psi(\tau) d \tau
$$

где $u_{1}(+\infty)=\lim _{t \rightarrow+\infty} u_{1}(t)$ и $\psi(t)$ положительная функция на $(0, \infty)$.

Далее, используя теорему 2, лемму 1 для невозрастающих функций, неравенство (7) и повторяя процесс доказательства теоремы 4, получим теорему 5.

Отметим, что теоремы 4 и 5 в случае, когда $u=u_{1}=1$, другим методом были доказаны в [4].

Автор благодарен профессору В. С. Гулиеву и академику А. Д. Гаджиеву за обсуждение полученных результатов.

\section{СПИСОК ЦИТИРОВАННОЙ ЛИТЕРАТУРЫ}

[1] A. P. Calderon, A. Zygmund, "On the existence of certain singular integrals", Acta. Math., 88 (1952), 85-139.

[2] L. Hormander, "Estimates for translation invariant operators in $L^{p}$ spaces", Acta. Math., 104 (1960), 93-140.

[3] И. М. Стейн, Сингулярные интеграль и дифференциальные свойства функиий, Мир, M., 1973.

[4] R. Trujillo-Gonzalez, "Weighted norm inequalities for singular integrals operators satisfying a variant of Hormander condition", Comment. Math. Univ. Carolinae 44, 1 (2003), $137-152$.

[5] D. J. Grubb, C. N. Moore, "A variant of Hormander condition for singular integrals", Colloq. Math., 73:2 (1997), 165-172.

[6] J. Garcia-Cuerva, J. L. Rubio de Francia, Weighted norm inequalities and related topics, North-Holland Math. Studies, 116, Amsterdam, 1985.

[7] D. K. Watson, "Weighted estimates for singular integrals via Fourier transform estimates", Duke Math. J., 60:2 (1990), 389-399.

[8] B. Muckenhoupt, "Weighted norm inequalities for Hardy maximal functions", Trans. Amer. Math. Soc., 165 (1972), 207-226.

[9] B. Muckenhoupt, "Hardy's inequality with weights", Studia Math., 44:1 (1972), 31-38.

[10] G. Talenti, "Asservazioni sopra and classe disuguaglianze", Rend. Sem. Math. e F is Milano, 1969, 171-185.

[11] G. Tomaselli, "A class of inequalities", Bull Unione Math. Italy, 2:2 (1969), 622-631.

[12] I. A. Aliev, A. D. Gadjiev, "Weighted estimates for multidimensional singular integrals generated by the generalized shift operators", Mat. sb., 183:9 (1992), 45-66.

[13] V.S. Guliyev, R.A. Bandaliyev, "Two-weight inequalities for integral operators in $L_{p^{-}}$ spaces of Banach-valued functions and their applications", Tr. Mat. Inst. Steklova, Ross. Akad. Nauk, 243 (2003), 194-212.

\section{Р. А. Бандалиев}

Поступила в редакцию

Институт математики и механики НАН Азербайджана 12.03 .2004

E-mail: szaman@yours.com 